\title{
Competition of strength and stress disorder in creep rupture
}

\author{
Zoltán Halász, Zsuzsa Danku, and Ferenc Kun* \\ Department of Theoretical Physics, University of Debrecen, P.O. Box 5, H-4010 Debrecen, Hungary
}

(Received 11 November 2011; published 25 January 2012)

\begin{abstract}
Based on a fiber bundle model of subcritical fracture with localized load sharing, we show that the interplay of threshold disorder and the inhomogeneous stress field gives rise to a rich dynamics with intriguing aspects. In the model, fibers fail either due to immediate breaking or to a slow damage process. When the disorder is strong, a large amount of damage occurs, which is randomly diffused over the system; however, for weak disorder, a single growing crack is formed, which proceeds in a large number of localized bursts. The microstructure of cracks is characterized by a power-law size distribution, which is analogous to percolation in the regime of diffusive damage; however, it becomes significantly steeper when a single crack dominates. Simulations showed that the size distribution of breaking bursts and of the waiting times in between have a power-law functional form with a load-dependent cutoff. The burst size exponent proved to be independent of the damage process; however, it strongly depends on the external load with a minimum value of 1.75 . The waiting time distribution is sensitive to the details of the damage process with an exponent decreasing from 2.0 to 1.4 as bursts get more and more localized to an advancing crack front.
\end{abstract}

DOI: 10.1103/PhysRevE.85.016116

PACS number(s): 46.50.+a, 62.20.M-, 64.60.av

\section{INTRODUCTION}

Subcritical fracture occurring under constant or periodic external loads represents an interesting scientific problem with a broad spectrum of technological applications. Experimental and theoretical studies revealed that a large variety of microscopic mechanisms contribute to the subcritical fracture process, from thermally activated microcrack nucleation through aging of material elements to viscoelastic relaxation [1-8].

Following the early works of Daniels and Coleman $[9,10]$, the fiber bundle model (FBM) became one of the most efficient theoretical approaches to subcritical fracture. In the FBM, the sample is composed of a parallel set of fibers with identical elastic properties but with stochastic failure thresholds [9-13]. By enhancing the single fibers by viscoelastic or plastic behavior, time-dependent rheology can be introduced into the model [14-21]. Several different rheological models have been considered in the framework of FBMs, which allowed an understanding of some aspects of damage enhanced creep (constant load) and fatigue fracture (periodic loading) [22-28]. The effect of thermal activation on the creep process at finite temperatures has also been captured in FBMs, either by introducing Gaussian-type fluctuations of the local stress [29-31] or assuming a Gaussian functional form for the failure probability of fibers [32].

Recently, we have introduced a different type of extension of the fiber bundle model to study the subcritical fracture of heterogeneous materials [25-28]. In the model, the fibers fail for two reasons. First, when the local load exceeds the mechanical strength of the fibers, they break immediately. Second, time dependence is introduced such that intact fibers undergo an aging process, in which they accumulate damage and break when the amount of damage exceeds a threshold value. The rate of damage accumulation is assumed to have a power-law dependence on the local load. We have carried out a detailed analytical and numerical study of the model and

*feri@dtp.atomki.hu showed that the competition of the two failure modes leads to a rich dynamics $[25,27,28]$. However, these studies were restricted to the mean-field limit of FBMs where no stress concentration can arise in the system. Due to the lack of spatial correlations, mean-field models cannot account for the growth of cracks and for the microstructure of damage relevant in realistic situations.

To overcome this limitation, in the present study we extend our FBM, introduced in Refs. [25,27,28], by considering localized load redistribution after fiber breakings, and investigate the effect of the strength of threshold disorder on the subcritical fracture process in the presence of an inhomogeneous stress field. The stress localization makes possible the growth of clusters of broken fibers, which represent cracks in the model. We show that the spatial structure of damage strongly depends on the amount of strength disorder and on the sensitivity of the aging process to the stress inhomogeneities. By varying the amount of disorder and the damage accumulation exponent, a transition is obtained from a phase where damage is homogeneously spread over the system, resulting in a simultaneous growth of a large number of cracks, to another phase where a single crack grows with a large number of localized bursts. We demonstrate that the existence of two phases has a strong effect on the behavior of the distribution of burst sizes and the waiting times between bursts.

\section{MODEL}

In the model, we consider a parallel bundle of fibers assembled on a square lattice. The fibers have a brittle response, i.e., they exhibit a linearly elastic behavior and fail abruptly when the load on them, $\sigma$, exceeds a critical value $\sigma_{t h}[25,27]$. The Young modulus of fibers $E$ is fixed at $E=1$, while the failure threshold $\sigma_{t h}$ is a random variable with a probability density function $g\left(\sigma_{t h}\right)$. In order to capture the aging of material elements subject to long time loading, we assume that intact fibers accumulate damage in the form of microcracks. The total amount of damage $c(t)$ of a fiber accumulated up to time 
$t$ can be obtained by integrating over the loading history of fibers,

$$
c(t)=a \int_{0}^{t} \sigma\left(t^{\prime}\right)^{\gamma} d t^{\prime}
$$

where the exponent $\gamma$ controls the rate of damage accumulation, while $a$ is a scale parameter. The fibers can only tolerate a limited amount of damage and break when $c(t)$ exceeds a threshold value $c_{t h}$, which is again a random variable with a probability density function $f\left(c_{t h}\right)$. Damage accumulation is the mechanism which introduces time in the model and leads to macroscopic failure of the bundle at any finite load.

\section{A. Breaking process of fiber bundles}

Under a constant external load $\sigma_{0}$, after each failure event the load of the broken fiber has to be overtaken by the remaining intact ones. In order to study the effect of stress inhomogeneities in the fracture process, we assume that the excess load is equally redistributed over the intact nearest neighbors of the failed fiber, i.e., at most, over four fibers on the square lattice. This localized load sharing (LLS) represents short-range interaction and leads to high stress concentrations around failed regions [33-38].

When the bundle is subject to a constant external load $\sigma_{0}$, the weak fibers with $\sigma_{t h}<\sigma_{0}$ break immediately and serve as nucleation centers for the growth of broken clusters. The load transferred to the nearest neighbors of broken fibers gives rise to further breakings. Consequently, when the system stabilizes itself after the initial breakings, the bundle contains small clusters of broken fibers randomly scattered over the system. Along the perimeter of clusters, there is a high stress concentration on the intact fibers. Increasing the value of the constant external load $\sigma_{0}$, a larger amount of initial breakings occurs, giving rise to larger clusters. It has been shown in Refs. $[36,37,39,40]$ that due to the high stress concentration, this LLS system cannot tolerate large broken clusters, and even in the limit $\sigma \rightarrow \sigma_{c}$, the size distribution of these initial clusters remains a rapidly decreasing exponential.

The time evolution of the system starts from this initial state with an inhomogeneous load distribution over the remaining intact fibers. As time elapses, the intact fibers accumulate damage and break slowly according to their damage thresholds, $c_{t h}^{i}(i=1, \ldots, N)$. After damage breakings, the load is again redistributed locally, further increasing the stress concentration along broken clusters. It is important to emphasize that the stress concentration enhances both the rate of damage accumulation according to Eq. (1) and the chance of immediate breaking in the vicinity of broken clusters. As a consequence, the fiber breakings become spatially correlated resulting in growing broken clusters, which represent growing cracks in the system.

The presence of stress concentrations also affects the temporal occurrence of local breaking events: We assume that the time scale of load redistribution $t_{r}$ is much shorter than the one of damage accumulation $t_{d}$, so that $t_{r} \ll t_{d} \simeq$ $1 / a \sigma_{0}^{\gamma}$ holds. After a number of $\Delta_{d}$ damage breakings, the resulting local load increments can be sufficient to induce an immediate breaking, which can then initiate an entire avalanche of breaking events. Hence, due to the separation of time scales of the two competing failure mechanisms of fibers, the slow damage sequences trigger bursts of immediate breakings, resulting in a jerky fracture process. Since the bursts are spatially correlated immediate breaking events, they represent growth steps of advancing cracks, while in the case of global load sharing, fiber breakings of avalanches are randomly scattered all over the system [27,33-35,37,40-42]. More details of the model construction, together with the analytic solution of the model in the limit of equal load sharing (ELS), can be found in Refs. [25-28].

\section{B. Competition of strength and stress disorder}

The aging process of fibers described by Eq. (1) becomes complicated due to the inhomogeneous stress field. It can be seen in Eq. (1) that over an infinitesimal time duration $d t$, the increment of fiber damage is

$$
\Delta c_{i}=a \sigma_{i}^{\gamma}(t) d t
$$

where $\sigma_{i}(t)$ is the local load of fiber $i(i=1, \ldots, N)$ at time $t$. It follows that in the case of local load sharing, the exponent $\gamma$ of damage accumulation controls the sensitivity of the system to the stress inhomogeneities: for $\gamma=0$, the damage accumulation process is completely independent of the local load $\sigma_{i}$ of fibers so that the sequence of damage breakings is fully controlled by the random damage thresholds $c_{t h}^{i}$. In damage sequences, the fibers break one by one in the increasing order of their damage thresholds, $c_{t h}^{i}(i=$ $1, \ldots, N)$. Assuming that the external load $\sigma_{0}$ is low enough so that most of the fibers break in damage sequences, it can easily be calculated that at $\gamma=0$, the lifetime $t_{f}$ of the system becomes independent of $\sigma_{0}$,

$$
t_{f}=\frac{c_{\text {max }}^{\max }}{a}
$$

where $c_{t h}^{\max }$ denotes the largest value of the damage thresholds. The consecutive fibers breaking in damage sequences are randomly scattered over the sample so that the spatial structure of damage in this limiting case can be described by percolation [43].

In the opposite limit of very large exponent $\gamma \rightarrow+\infty$, the system becomes very sensitive to the inhomogeneous stress distribution of intact fibers, i.e., the damage accumulation process becomes so fast that the inhomogeneous stress distribution dominates the failure process, while the random damage thresholds become irrelevant. The fiber that has the highest load initiates a spatially localized damage sequence, which proceeds by always breaking the fiber with the highest local load. Consequently, a single growing cluster emerges, which gradually covers the entire system. Intermediate values of the exponent $\gamma$ determine subsets of fibers where the amount of incremental damage $\Delta c_{i}$ is sufficient to generate damage breakings. Depending on the damage thresholds $c_{t h}^{i}$ of fibers, the position of consecutive damage breakings is not localized completely: damage breakings can switch from one place to another, giving rise to a large number of simultaneously growing cracks.

It follows from the above arguments that by varying the value of the exponent $\gamma$ of the damage accumulation law and the amount of disorder of the damage thresholds $f\left(c_{t h}\right)$, the 


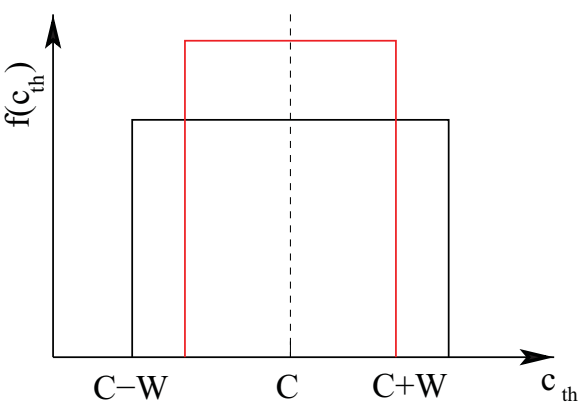

FIG. 1. (Color online) Disorder distribution of the damage thresholds $f\left(c_{t h}\right)$. By varying the width $W$ of the distribution, we can control the amount of disorder: the red (gray) curve represents a lower disorder than the black one. In the specific calculations, $C$ had the value $C=1$, so that $W$ was varied in the interval $0 \leqslant W \leqslant 1$.

subcritical system exhibits a transition from a regime where a single crack propagates until macroscopic failure, to another regime where damage is scattered all over the system leading to the simultaneous growth of a large number of cracks. In order to obtain quantitative insight into the nature of the transition, we consider a uniform distribution of breaking thresholds of fibers $\sigma_{t h}$ between 0 and 1 so that $g\left(\sigma_{t h}\right)=1$, with the cumulative distribution $G\left(\sigma_{t h}\right)=\sigma_{t h}$. For the damage thresholds $c_{t h}$, a uniform distribution is considered as well; however, with a controllable strength of disorder,

$$
f\left(c_{t h}\right)= \begin{cases}\frac{1}{2 W} & \text { for } \quad C-W \leqslant c_{t h} \leqslant C+W, \\ 0 & \text { elsewhere }\end{cases}
$$

where the average value of the damage thresholds is denoted by $C$, about which the threshold values $c_{t h}$ can vary over an interval of width $2 W$. The amount of disorder of damage thresholds $c_{t h}$ can be controlled by varying the width of the distribution $W$ in the range $0 \leqslant W \leqslant C$. Figure 1 illustrates the probability density $f\left(c_{t h}\right)$ of damage thresholds, where $C=1$ was used such that $0 \leqslant W \leqslant 1$ follows.

Based on the disorder distributions defined above, we can analytically estimate the parameter regimes which determine the appearance of single crack growth and diffusive damage. Let us assume that the external load $\sigma_{0}$ is low enough so that the immediate breaking of fibers does not trigger any bursts. In this case, when the time evolution of the system starts, only single broken fibers are present in the system for which $\sigma_{t h}^{i}<\sigma_{0}$ holds. In this state, due to the localized load redistribution, those fibers which do not have any broken neighbors have the remote load $\sigma_{0}$, while the neighbors of the broken ones have an elevated load value of $5 \sigma_{0} / 4$. When a fiber has a damage threshold $c_{t h}$, the time $t_{b}$ elapsed until the fiber breaks under a load $\sigma$ can be obtained from the damage law given by Eq. (1) as

$$
t_{b}\left(c_{t h}, \sigma\right)=\frac{c_{t h}}{a \sigma^{\gamma}} .
$$

Single crack growth can emerge if the next fiber to break due to damage is one of the overloaded fibers around the broken ones. In the worst case, this fiber has the highest possible damage threshold $C+W$, so that its time to break, $t_{b}\left(C+W, 5 \sigma_{0} / 4\right)$, should be compared to the breaking time of fibers with the

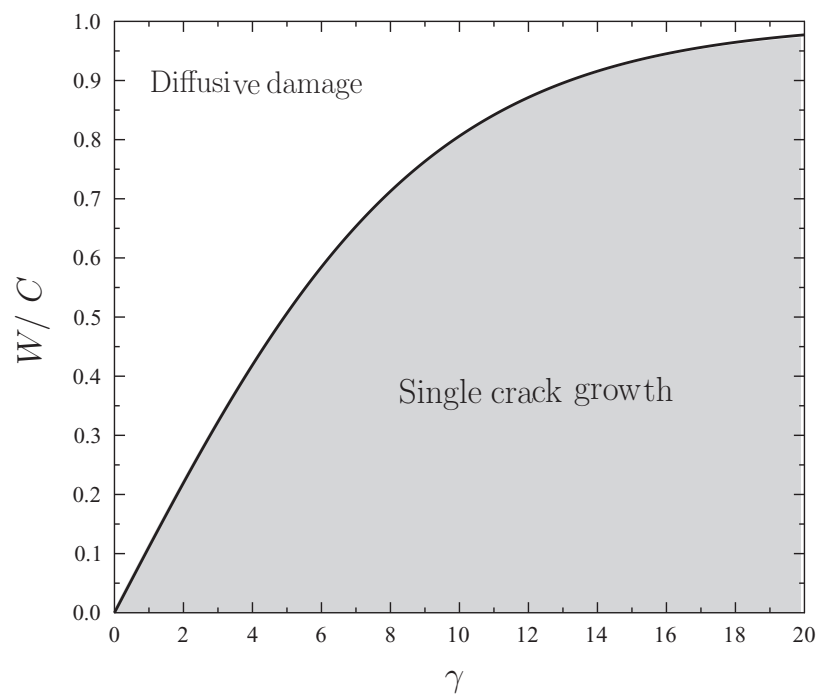

FIG. 2. Phase diagram of the system. The curve corresponding to Eq. (7) separates the regimes of single crack growth and diffusive damage.

background load having the lowest damage threshold, $t_{b}(C-$ $\left.W, \sigma_{0}\right)$.

It follows that the approximate condition of the emergence of a single growing crack is

$$
t_{b}\left(C+W, 5 \sigma_{0} / 4\right)<t_{b}\left(C-W, \sigma_{0}\right),
$$

which leads to a relation of the parameters characterizing the strength of disorder $W / C$ and the damage accumulation exponent $\gamma$ as

$$
\frac{W}{C}<\frac{\left(\frac{5}{4}\right)^{\gamma}-1}{\left(\frac{5}{4}\right)^{\gamma}+1} .
$$

Assuming equality in the above expression, the relation of $W / C$ and $\gamma$ is illustrated in Fig. 2. It can be seen in the figure that at low values of $\gamma$, even a small amount of disorder, i.e., $W / C \ll 1$, is enough to obtain diffusive damage spreading over the entire lattice, and simultaneously growing broken clusters. However, at high $\gamma$ exponents, single crack growth is expected up to a very large amount of disorder, i.e., $W / C \approx 1$.

\section{MACROSCOPIC EVOLUTION AND MICROSCOPIC STRUCTURE}

Computer simulations were carried out in order to study the time-dependent damage and fracture of the LLS fiber bundle under a constant external load $\sigma_{0}$. As we have discussed, slowly proceeding damage sequences can trigger bursts of immediate breakings, in which a large number of fibers break practically instantaneously. The final failure of the system occurs when a catastrophic burst emerges, i.e., a burst which does not stop until all intact fibers are swept away. First, we determined the fracture strength $\sigma_{c}$ of the bundle as the smallest load under which already the first avalanche leads to immediate catastrophic collapse. Under localized load sharing conditions, the fracture strength $\sigma_{c}$ of FBMs is known to have a logarithmic size effect [37]. In the present paper, quantitative results are 


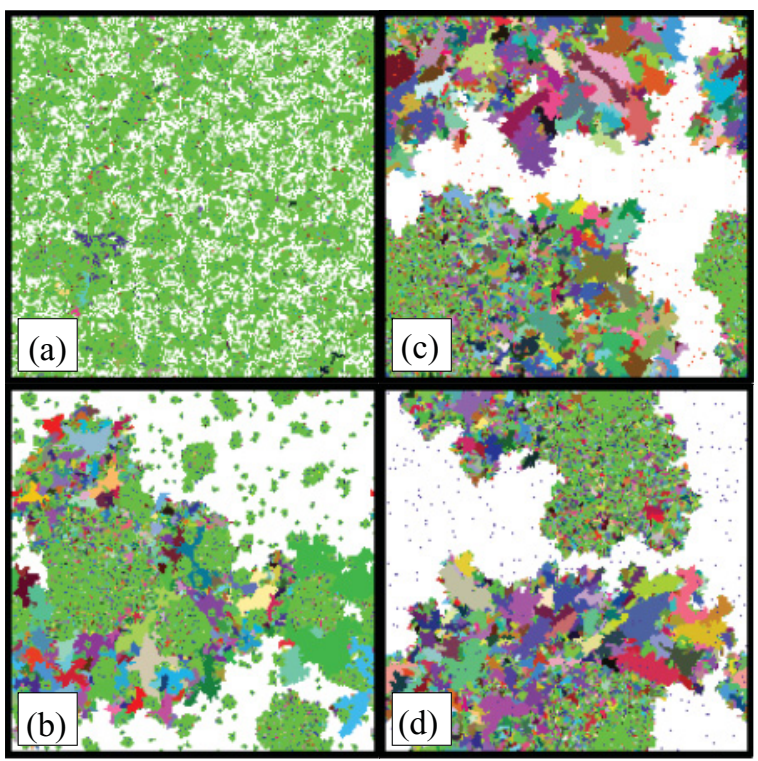

FIG. 3. (Color online) Cluster structure of broken fibers formed during the time evolution of a system of size $L=201$ for the same load, $\sigma_{0} / \sigma_{c}=0.084$, and amount of disorder, $W / C=0.2$, at different values of the $\gamma$ exponent: (a) 1.0, (b) 2.0, (c) 5.0, and (d) 8.0. Green color indicates the fibers that broke due to damage accumulation, while different colored spots represent broken fibers of bursts triggered by damage sequences. Periodic boundary conditions were used in both directions. (a) At low values of $\gamma$, most of the fibers break due to damage. (b) By increasing $\gamma$, damage sequences get spatially localized, which can trigger larger and larger bursts. (c), (d) At high $\gamma$, only a small amount of dispersed damage occurs, and practically a single large cluster is formed, which is a growing crack in the system.

presented at the system size $L=401$ for which the average critical stress was obtained numerically, i.e., $\sigma_{c}=0.149 \pm$ 0.0005 . Note that the corresponding fracture strength of FBMs with equal load sharing is $\sigma_{c}=0.25$ for the limit $N \rightarrow \infty$.

We analyzed the creep rupture process of the fiber bundle by computer simulations varying the load $\sigma_{0}$ in a broad range, i.e., $5 \times 10^{-4} \leqslant \sigma_{0} / \sigma_{c} \leqslant 5 \times 10^{-1}$. Figure 3 presents the last stable configuration of fiber bundles at the same value of the external load, $\sigma_{0} / \sigma_{c}=0.084$. For clarity, the amount of damage threshold disorder is fixed at $W / C=0.2$, and the value of the damage accumulation exponent $\gamma$ is varied. The results of Fig. 3 correspond to a horizontal line at the height $W / C=0.2$ in the phase space presented in Fig. 2. A relatively small system size is chosen for demonstration to be able to see the details of the microstructure. In Fig. 3, light green indicates fibers which break due to damage, while all other colors represent fibers which break in bursts of immediate failure. The colored spots show the spatial extent of these bursts. It can be observed in Fig. 3(a) that for $\gamma=1$, a large number of fibers break due to damage, until the emergence of the final catastrophic avalanche. Due to the low value of $\gamma$, the damage sequences are not spatially localized, hence, no high stress concentration can occur in the system, and, consequently, hardly any bursts are triggered. The value $\gamma=2$ in Fig. 3(b) falls practically on the phase boundary between single crack growth and diffusive damage (see also Fig. 2). It is very interesting to note that the large spots with colors different from light green indicate extended bursts of immediate breakings. Almost all of them belong to the same major cluster, i.e., these bursts are sudden advancements of a growing crack. Apart from the dominating cluster, there are smaller clusters of light green color grown by damage sequences. However, compared to Fig. 3(a), the difference is that all of these clusters were grown from small clusters of immediate failures generated in the initial state. This is the reason why all green spots contain a small cluster inside with another color different from green. Figures 3(c) and 3(d) belong to the phase of single crack growth with $\gamma=5$ and $\gamma=8$, hence, a dominating cluster emerges, which proceeds in a large number of localized bursts. Since in this phase the damage sequences are completely localized, outside the dominating cluster no damaged fibers can occur; all of the small clusters are just what remains of the initial dispersed breaking.

These computer simulation findings are in perfect agreement with our analytic results and completely support the existence of the two phases of the system. It is interesting to note that in spite of the microscopic complexity on the macroscale, the system presents a uniform behavior. The most important macroscopic characteristic quantity of the time evolution is the lifetime $t_{f}$ of the system. Simulations revealed that the average lifetime $\left\langle t_{f}\right\rangle$ follows the Basquin law [27,44], i.e., $\left\langle t_{f}\right\rangle$ decreases as a power law of the external load,

$$
\left\langle t_{f}\right\rangle \sim\left(\sigma_{0} / \sigma_{c}\right)^{-\gamma}
$$

The exponent coincides with the exponent of damage accumulation $\gamma$ similar to the behavior of ELS fiber bundles [27] [see Fig. 4(a)]. It can be observed in Fig. 4(a) that deviations from the Basquin law only occur in the vicinity of $\sigma_{c}$, both in the phase of diffusive damage $(\gamma=1)$ and single crack propagation $(\gamma=2,5)$.

\section{BURSTS DRIVEN BY SLOW DAMAGING}

The governing microscopic mechanism of the rupture process is that slow damage sequences trigger bursts of immediate breakings. The value of the constant load affects both the damage accumulation, through Eq. (1), and also the triggering of bursts. To clarify the effect of the external load on the microscopic dynamics of rupture, first we determined the average burst size $\left\langle\Delta_{a v}\right\rangle$ as a function of $\sigma_{0}$. It can be observed in Fig. 4(b) that in the phase of diffusive damage $(\gamma=1)$, the average burst size is practically constant and it has a low value over the entire load range. Since damage is randomly scattered in the system, no strong overloads can build up locally, and, hence, only small sized avalanches can pop up. The situation drastically changes already at the phase boundary $\gamma=2$. Damaging fibers form clusters, which results in high overloads and, hence, can trigger a considerable bursting activity. Under a constant external load in the fiber bundle, the burst size is determined by two competing mechanisms. Initially, at higher load values, the triggering becomes more efficient, giving rise to larger bursts. However, when the load gets too high, i.e., $\sigma_{0} \rightarrow \sigma_{c}$, so much overload will be concentrated on the boundary of growing avalanches that the propagating avalanche cannot stop anymore. Consequently, when approaching the macroscopic strength of the system $\sigma_{c}$, 

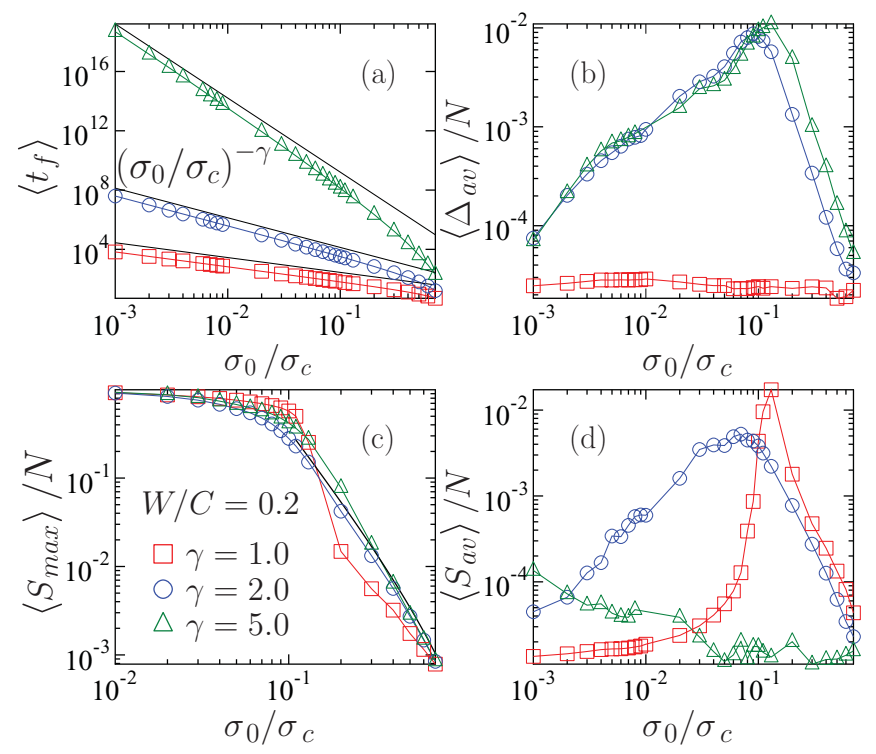

FIG. 4. (Color online) Average value of characteristic quantities of the bundle as a function of the external load, $\sigma_{0} / \sigma_{c}$, for three values of $\gamma$ with the amount of disorder $W / C=0.2$. In all of the subfigures, the same legend is used, which is presented in (c). (a) Average lifetime $\left\langle t_{f}\right\rangle$ of the bundle. Power-law behavior is obtained, showing the validity of the Basquin law. The slope of the straight lines is equal to the corresponding value of the damage accumulation exponent $\gamma$. (b) Average burst size. (c) Average size of the largest cluster of broken fibers. (d) Average cluster size. In (c), the thick line represents the fit obtained with Eq. (10).

the size of bursts after which the system still retains stability must be a decreasing function of $\sigma_{0}$. It can be observed in Fig. 4(b) that in agreement with the above argument, the average burst size $\left\langle\Delta_{a v}\right\rangle$ is strongly peaked inside the phase of single crack propagation $\gamma=5$, and even in the vicinity of the phase boundary $\gamma=2$. The position of the maximum, $\sigma_{0}^{m} / \sigma_{c} \approx 0.11$, proved to be practically independent of $\gamma$ when single crack propagation dominates. Well inside the regime of single crack propagation, all bursts are localized to an advancing crack front due to the dominance of stress inhomogeneities. This is demonstrated for $\gamma=8$ in Fig. 5, where the green damage sequences triggering the colorful bursts can also be observed.

The size distribution of bursts $P(\Delta)$ can be conceived as the distribution of the area of colored spots in Fig. 5. The distributions are presented in Fig. 6 for three $\gamma$ exponents selecting three load values, $\sigma_{0} / \sigma_{c}$, which lie at, before, and after the peak of $\left\langle\Delta_{a v}\right\rangle$ in Fig. 4(b). The most remarkable feature of the distributions is that at the peak load, they span several orders of magnitude, reaching the vicinity of the system size, $\Delta / N \approx 0.3$, and they exhibit a power-law behavior,

$$
P(\Delta) \sim \Delta^{-\xi},
$$

with an exponential cutoff. It has to be emphasized that the exponent is independent of $\gamma$ and its value $\xi=1.75$ is significantly smaller than the usual burst exponent of LLS bundles measured under quasistatic loading, $\xi \approx 4.0-4.5$ $[33,37,45]$. Away from the peak load $\sigma_{0}^{m} / \sigma_{0}$, the functional form of the distribution remains the same; however, the

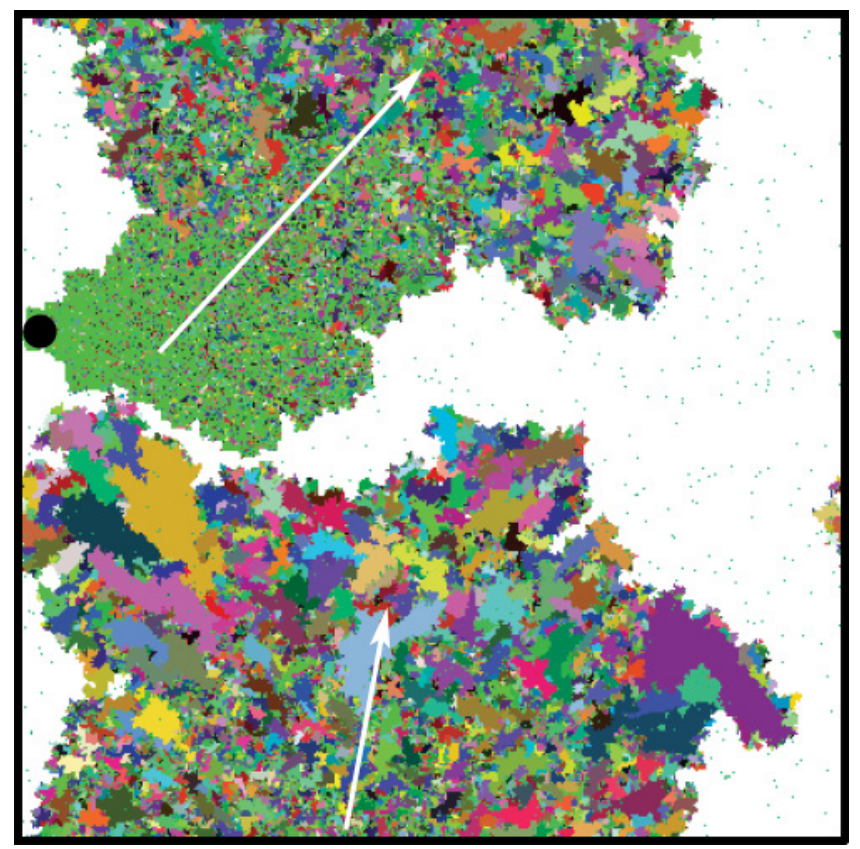

FIG. 5. (Color online) Spatial structure of bursts in a system of size $L=401$ with the parameter values $\gamma=8, W / C=0.2$, and $\sigma_{0} / \sigma_{c}=0.05$. Light green color indicates fibers broken due to damage accumulation. Spots of randomly selected colors represent bursts, i.e., simultaneously broken fibers, so that the extension of colored spots gives the burst size $\Delta$. The black circle on the left middle of the picture indicates the starting point of the crack and the white arrows show the direction of propagation (note the periodic boundary conditions in both directions).

cutoff shifts toward smaller burst sizes, in agreement with the behavior of the average burst size $\left\langle\Delta_{a v}\right\rangle$, and the value of the exponent $\xi$ increases to the vicinity of $\xi=2.5$. It is interesting to note that for equal load sharing, the burst size distribution of the creeping bundle has been found to

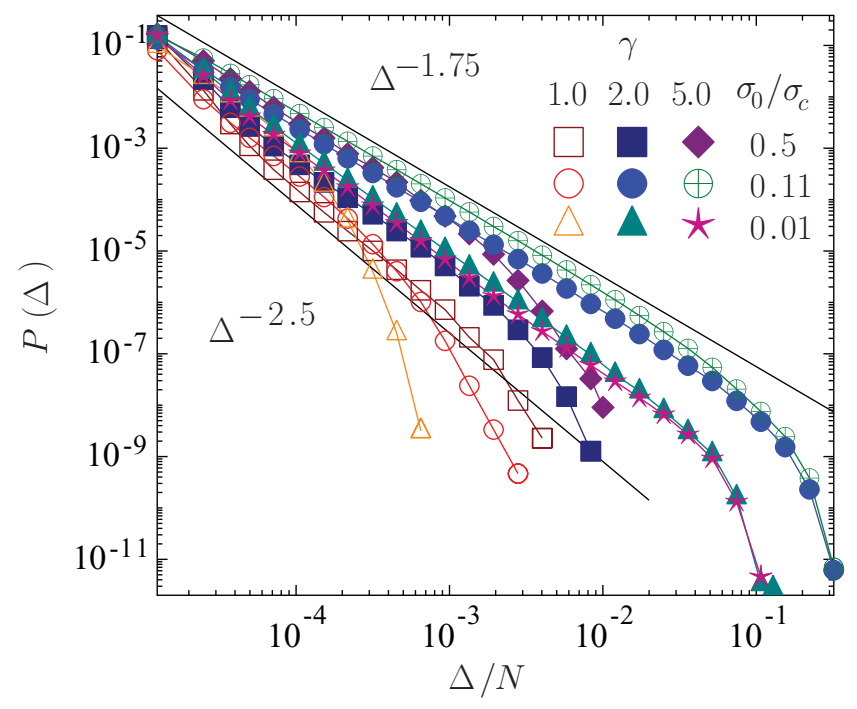

FIG. 6. (Color online) Burst size distributions for three values of $\gamma$ varying the load $\sigma_{0}$ in a broad range. It can be observed that at the load $\sigma_{0} / \sigma_{c}=0.11$, where the average burst size has a maximum, the exponent $\xi$ of the distribution $P(\Delta)$ has a minimum $\xi=1.75$. 
show practically the same behavior as under a quasistatically increasing external load [27]. Our simulations revealed that for localized load sharing, the breaking scenario of creep rupture becomes completely different from the quasistatic case $[33,37,45]$. Instead of the highly brittle abrupt rupture, the system smoothly approaches macroscopic failure due to the triggering effect of damage sequences.

\section{CLUSTER STRUCTURE}

The bursts of breaking events studied above characterize the temporal fluctuations of the time evolution of the system. Since load sharing is localized, broken fibers, irrespective of whether they break due to damage or due to immediate elastic response, form extended clusters in the system, which can be considered as cracks (see Figs. 3 and 5). For the quantitative characterization of the cluster structure, we determined the average size of the largest cluster $\left\langle S_{\max }\right\rangle$ in the last stable configuration of the system as a function of load $\sigma_{0}$. It can be observed in Fig. 4(c) that at low loads, when hardly any bursts are triggered, all broken fibers form a single connected cluster whose size is comparable to the system size, $\left\langle S_{\max }\right\rangle / N \approx 1$. However, approaching $\sigma_{c}$, a significantly lower fraction of fibers can break before catastrophic collapse, hence, the largest cluster decreases and a more complex cluster structure emerges. In the vicinity of the critical load, the value of $S_{\max }$ can be estimated based on the following argument: in a cluster of size $S$, the load of broken fibers $S \sigma_{0}$ is transferred to the intact ones along the cluster boundary. The number of perimeter fibers $n_{p}$ can be obtained as $n_{p} \sim \sqrt{S}$, and their load follows as $\sigma_{p} \approx \sigma_{0}(1+\sqrt{S})$. The cluster can grow until $\sigma_{p}$ falls below some critical value $\sigma_{p}<\sigma_{c}$ from which the size of the largest cluster as a function of $\sigma_{0}$ can be obtained as

$$
S_{\max } \sim\left[\left(\sigma_{c}-\sigma_{0}\right) / \sigma_{0}\right]^{\kappa},
$$

with $\kappa=2$. It can be seen in Fig. 4(c) that Eq. (10) provides a good quality fit of the numerical data for $\gamma=2$ and $\gamma=5$ when localization dominates. During fitting, the exponent $\kappa$ was used as a free parameter, which was then obtained to be $\kappa \approx 2.5$, in reasonable agreement with the above prediction.

The average size of clusters $S_{a v}$ was determined as the ratio of the second and first moments of cluster sizes $S_{a v}=$ $\sum_{i} S_{i}^{2} / \sum_{i} S_{i}$, skipping the largest cluster $S_{\max }$. Then, $S_{a v}$ was averaged over a large number of samples obtaining $\left\langle S_{a v}\right\rangle$, which is presented in Fig. 4(d). It is interesting to note that in the regime of diffusive damage, $\left\langle S_{a v}\right\rangle$ is sharply peaked at the load $\sigma_{0}^{m} / \sigma_{c} \approx 0.11$, which implies the emergence of a dominating cluster. At this load, the fraction of broken fibers is $p_{b} \approx 0.59$, which agrees well with the critical occupation probability of cite percolation on a square lattice [43]. The result demonstrates that the cluster structure of the phase of diffusive damage is analogous to percolation lattices due to the dominance of strength disorder [43]. When the damage is highly localized $(\gamma=5)$, i.e., inside the regime of single crack propagation, there is always a dominating cluster at any load which is significantly larger than any other clusters. Consequently, the corresponding curve of $\left\langle S_{a v}\right\rangle$ strongly fluctuates in Fig. 4(d) and it has small values. At the phase boundary, the cluster structure resembles both limiting cases, i.e., in Fig. 4(c), $\left\langle S_{a v}\right\rangle$ has a peak for $\gamma=2$; however, the

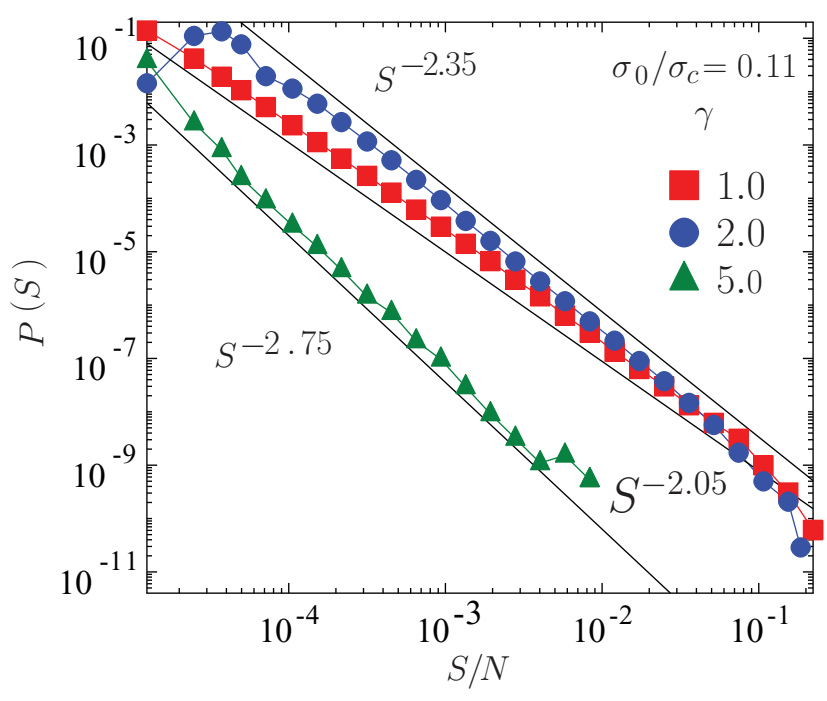

FIG. 7. (Color online) Cluster size distributions for three $\gamma$ values at the peak load of the average cluster size $\left\langle S_{a v}\right\rangle$. The exponent $\tau=2.05$ obtained for $\gamma=1$ is consistent with site percolation on a square lattice in two dimensions.

clusters emerge as a result of growth which is controlled by the stress localization.

The distribution of cluster sizes $P(S)$ is presented in Fig. 7 for the three $\gamma$ values considered. For clarity, the distributions are only shown at the load $\sigma^{m} / \sigma_{c} \approx 0.11$, where $\left\langle S_{a v}\right\rangle$ attains maximum. In all cases, power laws are obtained,

$$
P(S) \sim S^{-\tau},
$$

spanning over three to four orders of magnitude in a system of size $L=401$. It can be seen in the figure that the value of the exponent $\tau$ depends on $\gamma$ : In the diffusive regime, $\tau=$ $187 / 91$ is obtained numerically, supporting the percolation nature of the microstructure [43]. When stress inhomogeneities dominate the fracture process, the exponent gets higher, i.e., $\tau=2.35(\gamma=2)$ and $\tau=2.75(\gamma=5)$, which shows that the relative frequency of larger clusters gets smaller because a considerable fraction of broken fibers is involved in the emerging dominating cluster.

Note that in LLS FBMs subject to a quasistatically increasing external load, a completely different microstructure has been observed $[37,39,40]$ : In the last stable configuration of the system, the cluster size distribution $P(S)$ has an exponential form; furthermore, the size of the largest cluster does not scale with the system size $L$.

\section{WAITING TIMES BETWEEN BURSTS}

The time evolution of the system is mainly controlled by the damage accumulation process. The duration $T$ of damage sequences determines the waiting times between consecutive bursts. The lifetime $t_{f}$ of the creeping system can be obtained as the sum of waiting times,

$$
t_{f}=\sum_{i=1}^{K} T_{i},
$$




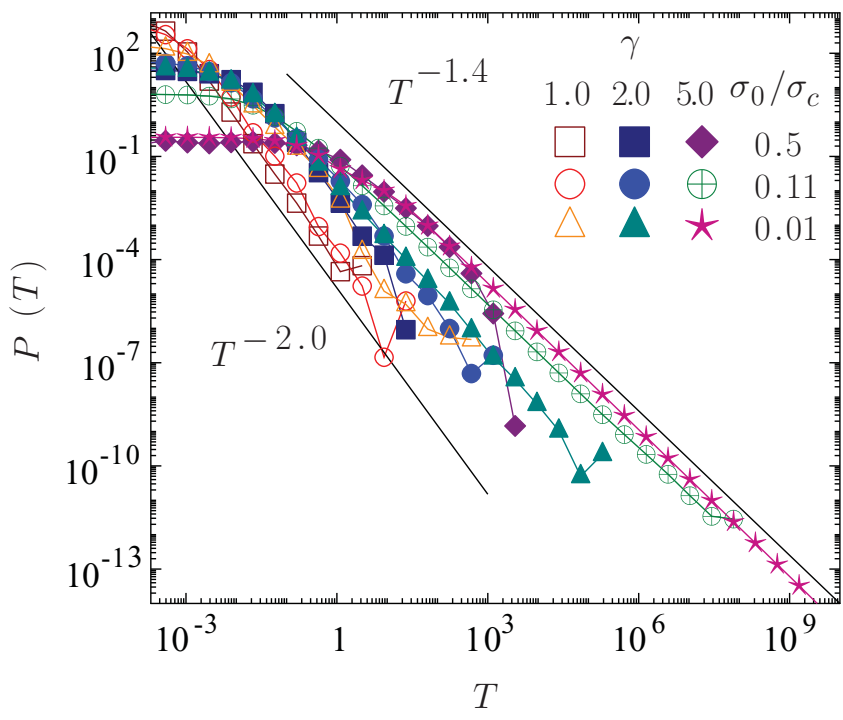

FIG. 8. (Color online) Distribution of waiting times $P(T)$ in a system of size $L=401$. A power-law functional form can be observed over eight orders of magnitude. The exponent of the power law $z$ is independent of the load $\sigma_{0}$; however, it changes from 1.4 to 2.0 as $\gamma$ is increased.

where $K$ denotes the total number of bursts up to failure. To characterize the time evolution of the system on the microlevel, we determined the probability distribution $P(T)$ of waiting times $T$. It can be seen in Fig. 8 that the waiting time distribution $P(T)$ follows a power-law behavior over a broad range of time $T$,

$$
P(T) \sim T^{-z},
$$

for all $\gamma$ and $\sigma_{0}$ considered. It is important to note that the exponent $z$ does not depend on $\sigma_{0}$; the load only controls the range of $T$ values: as $\sigma_{0}$ decreases, the maximum waiting time $T_{\max }$ scales as $T_{\max } \sim \sigma_{0}^{-\gamma}$, similarly to ELS bundles [27]. The most important feature of the distribution $P(T)$ is that the exponent $z$ strongly depends on the value of the damage accumulation exponent $\gamma$. In the phase of diffusive damage, the exponent $z$ tends to a limit value of $z=2$; however, in the phase of single crack propagation, a significantly lower value is obtained, i.e., $z=1.4$. The results indicate that when the stress concentration arising at the propagating crack front dominates the time evolution, large waiting times can frequently occur because the crack tip can be pinned by some strong fibers. However, when the quenched disorder of breaking thresholds controls the random nucleation of breakings, the waiting time exponent gets larger, i.e., $z=2$, since large waiting times may only rarely occur.

\section{DISCUSSION}

Based on a fiber bundle model of localized load sharing, we carried out a detailed investigation of the interplay of quenched structural disorder and of the inhomogeneous stress field during the creep rupture process of heterogeneous materials. The sensitivity of the system to the details of the stress field is controlled by the exponent of the law of damage accumulation. Assuming strongly localized load redistribution around failed fibers, we showed that the system has two phases, depending on the amount of strength disorder and on the damage accumulation exponent $\gamma$ : For high disorder and low values of $\gamma$, simultaneously growing cracks are spread homogeneously over the entire system. However, for low disorder and high $\gamma$ values, the damage gets localized to highly stressed regions, giving rise to a single growing crack. The jerky time evolution of the bundle is characterized by the size distribution of bursts and by the distribution of the waiting times elapsed in between. Both distributions proved to have a power-law behavior with a load-dependent cutoff. The most important outcome of the simulations is that the exponent of the burst size distribution proved to be independent of the details of the damage process such as the $\gamma$ exponent; however, it depends on the external load, which controls the efficiency of triggering. For the waiting time distributions, the opposite behavior is observed, i.e., the load only affects the cutoff of the distribution, while the exponent $z$ decreases from 2 to 1.4 as $\gamma$ is raised from 1 . We analyzed the microstructure of damage in the last stable configuration of the system just before catastrophic collapse. Simulations showed that under the dominance of disorder, the crack structure is analogous to percolation lattices, i.e., power-law distribution is obtained with a varying exponential cutoff. However, when a single crack propagates, the cluster size distribution becomes steeper.

Comparing the exponents of the burst size $\xi$ and waiting time distributions $z$ to the corresponding mean-field results, the exponent $\xi$ is significantly smaller, while $z$ is larger than the mean-field counterpart 2.5 and 1.0 , respectively [25,27]. The comparison indicates that when stress concentration dominates over disorder, the frequency of large bursts increases, but at the same time the failure process gets faster with shorter waiting times. The exponent of the waiting time distributions can be directly compared to experimental results on creeping systems. The creep experiment on paper provided waiting time exponents in the vicinity of 1.0 [46], which agrees well with the ELS results of our model [27]. The agreement implies that in this system, the long-range load redistribution rules the rupture process. For polyurethane foams, larger waiting time exponents were obtained experimentally ranging up to 1.4 [47]. A comparison to our model indicates the importance of localized bursts in the statistics.

\section{ACKNOWLEDGMENTS}

The work is supported by TAMOP-4.2.1/B-09/1/KONV2010-0007 project. The project implemented through the New Hungary Development Plan, co-financed by the European Social Fund and the European Regional Development Fund. F.K. acknowledges the support of OTKA K84157 and of the Bólyai János Foundation of the Hungarian Academy of Sciences. This work was supported by the European Commissions by the Complexity-NET pilot project LOCAT. The work is supported by the TAMOP-4.2.2/B-10/1-2010-0024 project. The project is co-financed by the European Union and the European Social Fund. 
[1] C. Maes, A. VanMoffaert, H. Frederix, and H. Strauven, Phys. Rev. B 57, 4987 (1998).

[2] H. Nechad, A. Helmstetter, R. E. Guerjouma, and D. Sornette, J. Mech. Phys. Solids 53, 1099 (2005).

[3] H. Nechad, A. Helmstetter, R. E. Guerjouma, and D. Sornette, Phys. Rev. Lett. 94, 045501 (2005).

[4] S. Deschanel, L. Vanel, G. Vigier, N. Godin, and S. Ciliberto, Int. J. Fract. 140, 87 (2006).

[5] J. Koivisto, J. Rosti, and M. J. Alava, Phys. Rev. Lett. 99, 145504 (2007).

[6] J. Davidsen, S. Stanchits, and G. Dresen, Phys. Rev. Lett. 98, 125502 (2007).

[7] S. Santucci, P. Cortet, S. Deschanel, L. Vanel, and S. Ciliberto, Europhys. Lett. 74, 595 (2006).

[8] S. Santucci, L. Vanel, and S. Ciliberto, Phys. Rev. Lett. 93, 095505 (2004).

[9] H. E. Daniels, Proc. R. Soc. London, Ser. A 183, 405 (1945).

[10] B. D. Coleman, J. Appl. Phys. 27, 862 (1956).

[11] M. Kloster, A. Hansen, and P. C. Hemmer, Phys. Rev. E 56, 2615 (1997).

[12] F. Kun, F. Raischel, R. C. Hidalgo, and H. J. Herrmann, in Modelling Critical and Catastrophic Phenomena in Geoscience: A Statistical Physics Approach, edited by P. Bhattacharyya and B. K. Chakrabarti, Lecture Notes in Physics (Springer-Verlag, Berlin, 2006), pp. 57-92.

[13] S. Pradhan, A. Hansen, and B. K. Chakrabarti, Rev. Mod. Phys. 82, 499 (2010).

[14] R. C. Hidalgo, F. Kun, and H. J. Herrmann, Phys. Rev. E 65, 032502 (2002).

[15] I. G. Main, Geophys. J. Int. 142, 151 (2000).

[16] T. Baxevanis, Europhys. Lett. 83, 46004 (2008).

[17] T. Baxevanis and T. Katsaounis, Phys. Rev. E 75, 046104 (2007).

[18] T. Baxevanis and T. Katsaounis, Eur. Phys. J. B 61, 153 (2008).

[19] F. Kun, R. C. Hidalgo, H. J. Herrmann, and K. F. Pal, Phys. Rev. E 67, 061802 (2003).

[20] F. Kun, Y. Moreno, R. C. Hidalgo, and H. J. Herrmann, Europhys. Lett. 63, 347 (2003).

[21] F. Raischel, F. Kun, and H. J. Herrmann, Phys. Rev. E 73, 066101 (2006).

[22] E. Rejovitzky and E. Altus, Int. J. Fatigue 33, 1235 (2011).

[23] A. Miksic, J. Koivisto, and M. Alava, J. Stat. Mech. (2011) P05002.

[24] E. A. Jagla, Phys. Rev. E 83, 046119 (2011).
[25] F. Kun, M. H. Costa, R. N. C. Filho, J. S. Andrade, J. B. Soares, and H. J. Herrmann, J. Stat. Mech. (2007) P02003.

[26] H. A. Carmona, F. Kun, J. S. Andrade, and H. J. Herrmann, Phys. Rev. E 75, 046115 (2007).

[27] F. Kun, H. A. Carmona, J. S. Andrade, and H. J. Herrmann, Phys. Rev. Lett. 100, 094301 (2008).

[28] F. Kun, Z. Halász, J. S. Andrade, and H. J. Herrmann, J. Stat. Mech. (2009) P01021.

[29] A. Guarino, A. Garcimartin, and S. Ciliberto, Europhys. Lett. 47, 456 (1999).

[30] A. Politi, S. Ciliberto, and R. Scorretti, Phys. Rev. E 66, 026107 (2002).

[31] S. Roux, Phys. Rev. E 62, 6164 (2000).

[32] S. Pradhan and B. K. Chakrabarti, Phys. Rev. E 67, 046124 (2003).

[33] A. Hansen and P. C. Hemmer, Phys. Lett. A 184, 394 (1994).

[34] W. I. Newman and S. L. Phoenix, Phys. Rev. E 63, 021507 (2001).

[35] S. L. Phoenix and W. I. Newman, Phys. Rev. E 80, 066115 (2009).

[36] F. Kun, S. Zapperi, and H. J. Herrmann, Eur. Phys. J. B 17, 269 (2000).

[37] R. C. Hidalgo, Y. Moreno, F. Kun, and H. J. Herrmann, Phys. Rev. E 65, 046148 (2002).

[38] F. Raischel, F. Kun, and H. J. Herrmann, Phys. Rev. E 74, 035104 (2006).

[39] S. Zapperi, P. Ray, H. E. Stanley, and A. Vespignani, Physica A 270, 57 (1999).

[40] S. Zapperi, P. Ray, H. E. Stanley, and A. Vespignani, Phys. Rev. Lett. 78, 1408 (1997).

[41] S. Pradhan, B. K. Chakrabarti, and A. Hansen, Phys. Rev. E 71, 036149 (2005).

[42] J. V. Andersen, D. Sornette, and K. Leung, Phys. Rev. Lett. 78, 2140 (1997).

[43] D. Stauffer and A. Aharony, Introduction to Percolation Theory (Taylor \& Francis, London, 1992).

[44] O. H. Basquin, Proceedings, American Society for Testing and Materials, ASTEA 10, 625 (1910).

[45] F. Kun and H. J. Herrmann, J. Mater. Sci. 35, 4685 (2000).

[46] L. I. Salminen, A. I. Tolvanen, and M. J. Alava, Phys. Rev. Lett. 89, 185503 (2002).

[47] S. Deschanel, L. Vanel, N. Godin, and S. Ciliberto, J. Stat. Mech. (2009) P01018. 